\title{
Validating International Classification of Disease 10th Revision Algorithms for Identifying Influenza and Respiratory Syncytial Virus Hospitalizations
}

\author{
Mackenzie Hamilton ${ }^{1,2}$, Andrew Calzavara ${ }^{1}$, Scott D Emerson ${ }^{1}$ and Jeffrey C Kwong ${ }^{1,2,3,4,5,6}$ \\ 1 ICES, Toronto, Ontario, Canada \\ 2 Dalla Lana School of Public Health, University of Toronto, Toronto, Canada \\ 3 Public Health Ontario, Toronto, Ontario, Canada \\ 4 Department of Family and Community Medicine, University of Toronto, Toronto, Canada \\ 5 University Health Network, Toronto, Canada \\ 6 Centre for Vaccine Preventable Diseases, University of Toronto, Toronto, Ontario, Canada \\ E-mail: jeff.kwong@utoronto.ca
}

Received October 28, 2020

Accepted for publication December 23, 2020

Published online September 26, 2021

\begin{abstract}
Introduction: Routinely collected health administrative data can be used to efficiently assess disease burden in large populations, but it is important to evaluate the validity of these data. The objective of this study was to develop and validate International Classification of Disease 10PthP revision (ICD -10) algorithms that identify laboratory-confirmed influenza or laboratory-confirmed respiratory syncytial virus (RSV) hospitalizations using population-based health administrative data from Ontario, Canada.
\end{abstract}

Methods: Influenza and RSV laboratory data from the 2014-15 through to 2017-18 respiratory virus seasons were obtained from the Ontario Laboratories Information System (OLIS) and were linked to hospital discharge abstract data to generate influenza and RSV reference cohorts. These reference cohorts were used to assess the sensitivity, specificity, positive predictive value (PPV) and negative predictive value (NPV) of the ICD-10 algorithms. To minimize misclassification in future studies, we prioritized specificity and PPV in selecting top-performing algorithms.

Results: 83,638 and 61,117 hospitalized patients were included in the influenza and RSV reference cohorts, respectively. The best influenza algorithm had a sensitivity of $73 \%$ (95\% CI $72 \%$ to $74 \%$ ), specificity of $99 \%$ (95\% CI 99\% to 99\%), PPV of 94\% (95\% CI $94 \%$ to $95 \%$ ), and NPV of $94 \%$ (95\% CI $94 \%$ to $95 \%$ ). The best RSV algorithm had a sensitivity of $69 \%$ (95\% CI $68 \%$ to $70 \%$ ), specificity of $99 \%$ (95\% CI $99 \%$ to $99 \%$ ), PPV of $91 \%$ (95\% CI $90 \%$ to $91 \%$ ) and NPV of $97 \%$ (95\% CI $97 \%$ to $97 \%)$.

Conclusion: We identified two highly specific algorithms that best ascertain patients hospitalized with influenza or RSV. These algorithms may be applied to hospitalized patients if data on laboratory tests are not available, and will thereby improve the power of future epidemiologic studies of influenza, RSV, and potentially other severe acute respiratory infections. 